\title{
Numerical Analysis of Solid Materials Transport in a Rotary Dryer
}

\author{
Miao-Ling $\mathrm{LI}^{\mathrm{a}}$, Yong-Yu YAO ${ }^{\mathrm{b}}$, Hong-Xia ZHAOc \\ School of Machnical Engineering, Luoyang Institute of Science and Technology, 471023, Luoyang, \\ China \\ amiaolingli1970@126.com, byaoyy72@163.com,clysunshine27@163.com
}

Keywords: Rotary dryer, Three-dimensional model, Material transportation, Modeling, Residence times, Numerical analysis.

\begin{abstract}
The throwing motion of materials in the rotary dryer is the key step to dry off. An estimation model of solid material transport was established. The motion rule about the lifted materials was studied. The numerical model was proposed according to the space motion of materials and mathematical calculation. And the formulas of residence time were deduced considering the weighted value of the holdup within the lifting plates. The proposed estimation models were determined by considering the air drag on the materials in the drum, as well as the inclination angle, the rotation speed and the filling coefficient of a drum. The calculation was compared with the experimental values from the industrial production example. A coefficient 0.9 is adapted to correct the theoretical residence time. The calculation formula of residence times was with a clear and definite physical meaning. The proposed model generated accurate estimations.
\end{abstract}

\section{Introduction}

A rotary dryer is mainly composed of a slightly inclined rotary drum, on the inner wall of which a series of lifting plates are fitted. The machine has the advantages of simple structure, high material adaptability and low operation costs. It is widely used for control of moisture content of solid materials in a range of industries, such as cement, mine, chemical industry, compound fertilizer, etc. But thermal efficiency of the rotary drying equipment is not enough high. Considering that material movement in the rotary dryer is a key determinant of drying, there is a need for the research on it. Mathematical analysis has been proved to be an effective way of solving some complex practical problems [1]. Building mathematical model of solid materials movement in a rotary dryer has important significance for optimizing the structure of lifting plates and improving the heat transfer efficiency. The work is expected helpful to promote the application and development of the rotary drying equipment.

The solids movement in a rotary drum can be divided into two major parts. One is space falling movement of materials lifted by the lifting plates. And the other is rolling motion of superficial materials in the lifting plates. The materials to be dried are lifted repeatedly and thrown down to fall through heat gas flow, which makes the material form even curtain and enhances heat exchange sufficiency. So, the falling movement is most important for drying materials. This movement is closely related to the structure of lifting plates [2]. Meanwhile, the movement of materials along the cylinder axial direction is rather complicated owing to the complex action resulted from the slope of the rotary drum, the force of the drying media to materials and some other factors, all of which have a direct influence on the residence time of materials in the rotary drum [3].

At present, the research on modeling method of the materials movement in the rotary dryer is usually based on the spreading model of materials carried by the lifting plates and their residence time model in the rotary drum. There have been some valuable research results [4-6]. But most of those rely on the empirical formula with const coefficients, whose physical meaning is not clear. This work is based on the falling mechanism of materials through the moving gas stream space. The main purpose is to establish the numerical analysis model of materials movement and to derive the formula of the residence time with the weighted value of the holdup within the lifting plates. The conclusions of the research will be verified in the industrial production. 


\section{Modeling of Material Motion in the Dryer Shell}

As shown in Fig. 1, a rotary dryer is installed in slope. The tilt angle is $\lambda$, the radius of the rotary drum is $R$, the rotating angular velocity is $\omega$ and the rotating radius of the end of the lifting plates is $r$.

Let's suppose that the material particles only keep on moving in a circular without rolling along the drum wall at the lifting stage. At one instant time $t$, the particles at the point $P_{0}$ are thrown with the initial velocity $V_{0}=\omega r$. Based on the coordinate system shown in Fig.1, there is the movement balance equation

$$
\sum F=m \frac{d^{2} r}{d t^{2}}
$$

Projections to each coordinate axis are here, $\mathrm{m}$ is the material mass (unit:

$\mathrm{kg}$ ); $\lambda$ is the tilt angle of the drum (unit: $\left.{ }^{\circ}\right)$; $\mathrm{G}$ is the gravity acceleration $(9.8 \mathrm{~m} / \mathrm{s} 2)$; $\mathrm{F}$ is the push force of drying gas flow (unit: $\mathrm{N}$ ). $\mathrm{F}$ is negative when the material flow is in reverse direction to the drying gas flow.

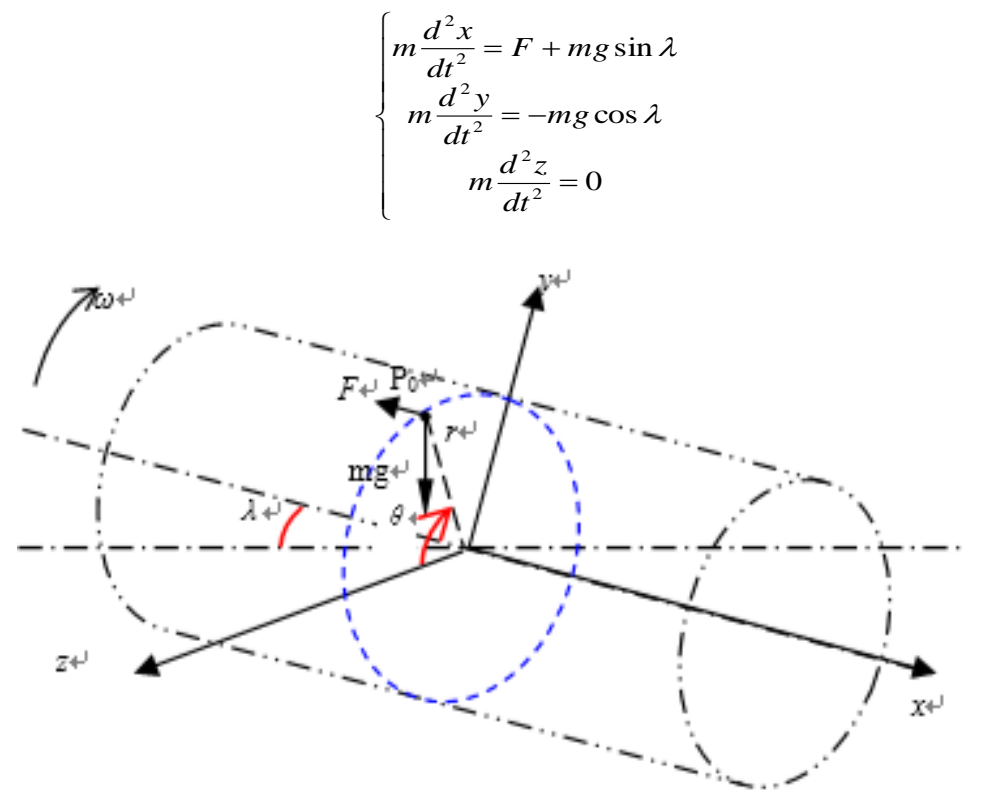

Fig.1 Diagram of material stress states in a rotary dryer

As shown in Fig.1, $\theta$ is defined as the angle of the point $P_{0}$ to the $+Z$ axis direction. Supposed that the point $P_{0}$ is the beginning position of throwing a particle and this moment is $t=0$, the initial conditions of material movement are defined: $\frac{d x}{d t}=0, \frac{d y}{d t}=r \omega \cos \theta, \frac{d z}{d t}=-r \omega \sin \theta$ and $x=0, y=r \sin \theta$, $z=r \cos \theta$. From Eq. (1), getting the following solution.

$$
\left\{\begin{array}{c}
x=\frac{1}{2 m}(F+m g \sin \lambda) t^{2} \\
y=-\frac{1}{2} g t^{2} \cos \lambda+r \omega t \cos \theta+r \sin \theta \\
z=-r \omega t \sin \theta+r \cos \theta
\end{array}\right.
$$

A falling cycle is finished when the material falls down to the circle of the end of the lifting plates. At the moment, there is $y^{2}+z^{2}=r^{2}$, combine with Eq.2, the falling time of the materials from the point $P_{0}$ can be obtained.

$$
t_{\text {throwing }}(\theta)=\frac{2 r \omega \cos \theta+2 \sqrt{r g \sin \theta \cos \lambda-r^{2} \omega^{2} \sin ^{2} \theta}}{g \cos \lambda}
$$

From Eq.3, it is observed that the falling time of the material particle is a function of position angle of the lifting plate.

Ideally, the lifting plate begins spreading materials at $\theta=0^{\circ}$ shown in Fig.1. And all materials should be thrown out till $\theta=180^{\circ}$. For example, the circle radius of the end of the lifting plates is 
$1.3 \mathrm{~m}$. The material spatial movement in a rotating cycle is shown in fig. 2 . The thick blue solid lines represent the falling tracks of material particles, and the thin red line represents the rotary circumference of the end of the lifting plates. Fig.2(a) is the three-dimensional falling trajectories of material particles, and Fig.2(b) is the projection of the trajectorys on the rotary circular crosssection (YZ). This is the ideal distribution of the materials in the rotary drum, i.e. a homogeneous material curtain is formed in the whole cylinder body.

It can be known from Fig.2 that the placement of the dropped material can be expressed as the angle $\theta_{0}$ (unit: ${ }^{\circ}$ )

$$
\theta_{0}=\left\{\begin{array}{cc}
180^{\circ}-\arctan \left(\frac{y}{z}\right), & \text { when } z \leq 0 \\
-\arctan \left(\frac{y}{z}\right), & \text { when } z>0
\end{array}\right.
$$

As shown in Fig. 3, when a rotary dryer runs well, the materials are stirred up from the drum bottom by the lifting plates. Along with the drum rotating, the lifting plates are rising stage by stage and the materials piled up in them are continuously rolling to the wing endpoints. Then, by the action of gravity and push force from drying gas flow, the materials start to fall off from the wing endpoints at a certain speed and form a continuous parabolic material curtain. At the same time, the materials held in the lifting plates reduce.
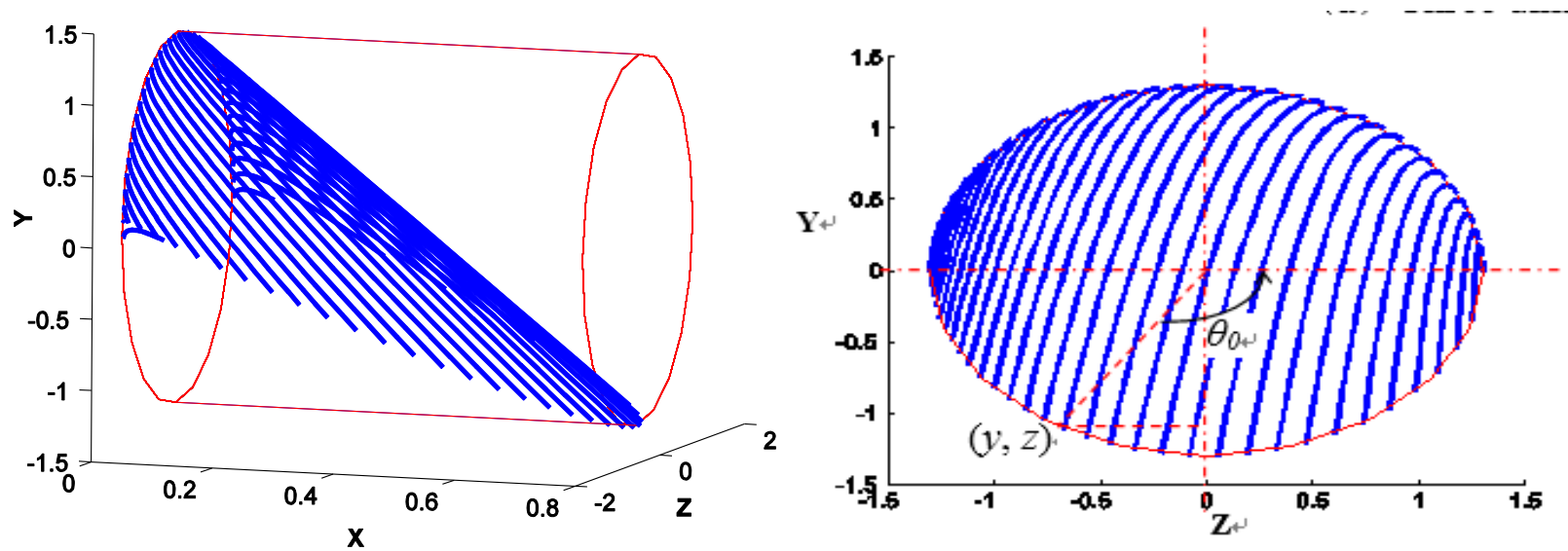

(a)Three dimensional motion trajectory

(b) Trajectory projection on circular cross section(YZ)

Figure 2. Schematic diagram of the movement track of the solid materials

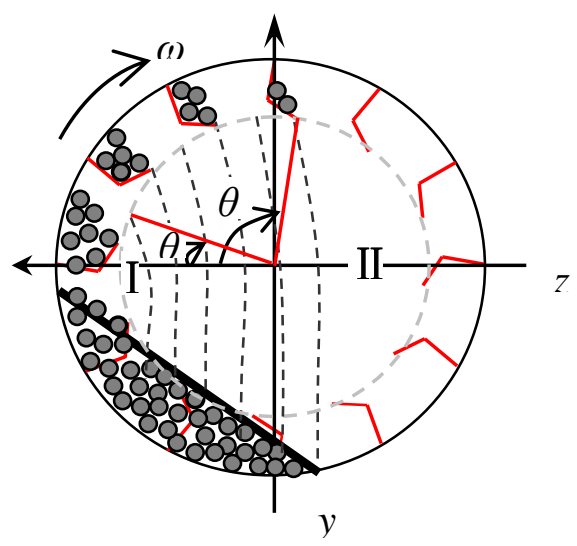

Figure 3. Holdup of the lifting plates and material motion states in a rotary drum

The distribution and uniformity of the material curtain is the main judgment parameter for dryer performance [7]. The characteristics of raw materials, the shape and installation angle of lifting plates, the rotary speed of the machine and other factors affect the falling status of the materials. In 
fact, when a lifting plate is over in the horizontal plane shown in the Fig.3. the materials held in it still have not started to fall down, resulting in the "wind tunnel" I often found in practical applications; when the lifting plate rotates but not to get the other side of the horizontal plane, the lifted materials have fallen down entirely, forming the "wind tunnel" II. The size of "wind tunnel" is determined by the space distribution of the lifted material between the ending lifting angle $\theta_{2}$ and the starting lifting angle $\theta_{1}$. The "wind tunnel" causes the flowing heat gas forming short circuit and decreases heat transfer efficiency.

\section{Numerical Analysis to Axial Movement and Residence Time of Materials}

When the dryer runs, the materials are continuously raised by the lifting plates, and then thrown down. Because the dryer slopes slightly, the particles have the axial movement toward the discharge end until they fall to the bottom of rotating shell. A cycle of lifting and throwing materials is completed. This is a repetitive movement process [8]. Let $W\left(\theta_{1}\right)$ express the maximum holding amount of a single lifting plate. If the lifting plate rotates through $\Delta \theta$, there is $\Delta W(\theta)$ material thrown. When the thrown materials from the lifting plate are considered as the weight, the average movement distance of the thrown materials along the cylinder axial is expressed as $\bar{x}_{\text {throwing }}$ (unit: s).

$$
\bar{x}_{\text {throwing }}=\frac{\sum_{\theta_{1}}^{\theta_{2}} \Delta W(\theta) x(\theta)}{W\left(\theta_{1}\right)}
$$

The mean time of throwing materials is expressed as $\bar{t}_{\text {throwing }}$ (unit: s).

$$
\bar{t}_{\text {throwing }}=\frac{\sum_{\theta_{1}}^{\theta_{2}} \Delta W(\theta) t_{\text {throwing }}(\theta)}{W\left(\theta_{1}\right)}
$$

The materials thrown down to the bottom of the dryer moves subsequently with the cylinder shell and is lifted again, until it is dropped. If the speed of the dryer is expressed as $n$ (unit: $\mathrm{r} / \mathrm{min}$ ), the average time for lifting materials along with the rotating cylinder is expressed as $\bar{t}_{\text {lifting }}$ (unit: s).

$$
\bar{t}_{\text {lifing }}=\frac{\sum_{\theta_{1}}^{\theta_{2}} \Delta W(\theta)\left[\theta+\theta_{0}(\theta)\right]}{6 n W\left(\theta_{1}\right)}
$$

If the rotating angular velocity is $\omega$ and the rotating radius of the end of the lifting plates is $r$.

Meanwhile, the average movement distance of the lifted materials along the cylinder axial is expressed as $\bar{x}_{\text {lifting }}$ (unit: $\mathrm{m}$ ) when the tilt angle and the radius of the rotary drum is $\lambda$ and $R$, respectively.

$$
\bar{x}_{\text {lifing }}=\frac{\sum_{\theta_{1}}^{\theta_{2}} \Delta W(\theta)\left[\theta+\theta_{0}(\theta)\right]}{180 W\left(\theta_{1}\right)} R \tan \lambda
$$

After the number and installation way of the lifting plates is defined, the duration of material movement and its axial displacement in the different stages are calculated respectively. They are summed into the residence time and the total displacement within the drum.

If the length of the rotary cylinder is $L$, the lifted times of the materials along the total length is

$$
c=\frac{L}{\bar{x}_{\text {throwing }}+\bar{x}_{\text {lifting }}}
$$

During a cycle, the average residence time of the materials is

$$
\bar{t}=\bar{t}_{\text {lifting }}+\bar{t}_{\text {throwing }}
$$

So, the total residence time of the materials in the rotating shell is

$$
T=c \bar{t}
$$

At any instant time $t$, the axial position of the materials in the rotating cylinder body is 


$$
X=\frac{t}{t}\left(\bar{x}_{\text {throwing }}+\bar{x}_{\text {lifting }}\right)
$$

\section{Application and Analysis}

A $\varphi 2.6 \times 20 \mathrm{~m}$ direct heating counter-current rotary drying machine is employed in a cement plant. Its productivity is $9500 \mathrm{~kg} / \mathrm{h}$. The drying particles are cement raw materials with average grain diameter $10 \mathrm{~mm}$, particle density $2 \times 10^{3} \mathrm{~kg} / \mathrm{m}^{3}$, packing density $0.8 \times 10^{3} \mathrm{~kg} / \mathrm{m}^{3}$, and repose angle $35^{\circ}$. The drying medium is hot air flow, its density $0.98 \mathrm{~kg} / \mathrm{m}^{3}$, the flow velocity $2 \mathrm{~m} / \mathrm{s}$, hot air viscosity about $1.2 \times 10^{-5} \mathrm{~m}^{2} / \mathrm{s}$. The cylinder body is inclined to the horizontal with angle $5^{\circ}$. And there are the uniformly distributed L-type lifting plates. The length and the included angle of both sections of a lifting plate are $0.26 \mathrm{~m}, 0.13 \mathrm{~m}$ and $90^{\circ}$. Its installation angle is $80^{\circ}$.

When the dryer works in the normal load, the volume filling coefficient is $15 \%$ and the rotation speed is $4 \mathrm{r} / \mathrm{min}$. The moisture contents of the fed raw materials and the dried products are measured, $8 \%$ and $0.5 \%$, respectively.

According the current state of the lifting plates in the drum, the theoretical starting lifting angle is $0^{\circ}$ and the theoretical terminate lifting angle is $120^{\circ}$. The movement parameters can be calculated on the basis of the founded numerical model. The results are as follows.

The moving distance of the materials raised by one lifting plate along the axis of the cylinder is $0.125 \mathrm{~m}$. The lifting time of the materials in the shell is 160 . The lifting period of the material is about 4.6s. The retention time of the material in the dryer shell is about $12 \mathrm{~min}$. The result are greater than the value measured in the production field by $1 \sim 1.5 \mathrm{~min}$. There are two reasons are considered through the detailed field observation and analysis:

(1)When the materials are throwing down, there are collisions between them. This increases drop time;

(2)The materials fall down to the bottom of the shell and contact the surface of the retention materials. Due to inertia, the fallen materials have the trend of rolling to the opposite direction of the shell rotation. This delays the begin time of the circumferential motion.

Therefore, it is more in line with the actual situation when Equ. (10) is considered to be multiplied by a coefficient of 0.9 .

In order to further verify the forementioned formulas, the calculated residence time is compared with the results from the formulas in the references [1,5,9]. The reference [1] gave the retention time (unit: $\min$ ) with the empirical formula

$$
\tau=\frac{1.77 \sqrt{\alpha L}}{D n \lambda} .
$$

here: $\alpha$ is material repose angle $\left(^{\circ}\right), L$ is barrel length $(\mathrm{m}), D$ is cylinder diameter $(\mathrm{m}), n$ is cylinder speed $(\mathrm{r} / \mathrm{min}), \lambda$ is cylinder fixing tilt angle.

According to $\mathrm{Eq}(13)$, the material retention time is calculated about $8.6 \mathrm{~min}$. But the empirical formula was acquired for the materials with uniform particles in the dryer shell without any lifting plate and retaining ring. The test conditions are very different with the practical situation. In an industry dryer, lifting plates can slow down material moving speeds, and increase the residence time. So the calculated result of the Equ.(13) needs to be corrected using the empirical coefficient.

In reference [5,9], the retention time (unit: $\mathrm{min}$ ) is given in the Equ. (14).

$$
\tau=\frac{0.3344 L}{\tan \lambda n^{0.9} D} \pm 0.6085 \frac{L G_{q}}{d^{0.5} G_{w}}
$$

here: $d$ is particle diameter $(\mu \mathrm{m}), G \mathrm{q}$ is gas mass velocity $\left[\mathrm{kg} /\left(\mathrm{m}^{2} \cdot \mathrm{s}\right)\right], G \mathrm{w}$ is material mass velocity $\left[\mathrm{kg} /\left(\mathrm{m}^{2} \cdot \mathrm{s}\right)\right]$, the meaning of the other symbols is the same as those in Equ. (13). "+" is taken in the countercurrent, that is gas direction is consistent with materials movement. "." in the downstream.

According to Eq. (14), the material retention time is $10.7 \mathrm{~min}$. The researchers would take cylinder rotation speed, rotating shell inclination, direction and rate of materials and gas flow, 
drying machine production, and other factors into consideration. This formula comprehensively reflects the motion state of materials. But, the application of the formula is limited because of its physics significance is ambiguous and its empirical coefficient is excessive.

Using the modeling method in this paper, the calculated result is close to that of the formula (14). The difference is $1.3 \mathrm{~min}$. When the Equ. (11) is multiplied by the correction coefficient 0.9 , the results are almost similar. This further verifies the validity of the formula discussed in this paper. Furthermore, the physical meaning of the derived formula is clear.

\section{Conclusions}

In the rotary dryer, the throwing motion of materials lets those contact to hot air fully. It is the key step to dry off. This paper studies the material motion in the rotary dryer, establishes the kinematics model and the numerical analysis model of the residence time. After the materials in the axial and circumferential motion are analyzed, the theoretical material movement route is obtained. The material motion state in the dryer shell is displayed by the three-dimensional image. The established model synthesizes cylinder inclination, drying medium thrust, dryer rotation speed, cylinder filling coefficient and other factors. Through the practical application example, and the experience formula contrast, the derived formula is reasonable, and its physical meaning is clear.

\section{Acknowledgments}

This work is supported by the Science and Technology Innovation Plan of National Building Materials Industry, China (No. 2015-M1-2).

\section{Reference}

1. G. W. Liu, Design of drying equipment (Mechanical Industry Press, Beijing, 2009)

2. T. P. Li and S. J. Cai, Cement Guide for New Epoch, 2(2001)

3. Sh. Shahhosseini, M.T. Sadeghi and H. R. Golsefatan, Braz. J. Chem. Eng. 7, 2(2010)

4. G. J. Zhao, J. Wu and F. J. Yin, C. Sheng and Z. J. Zhang, Chem. Eng. \& Mach. 36, 3(2009)

5. M. H. Lisboa, D. S. Vitorino, W. B. Delaiba, J. R. D. Finzer and M. A. S. Barrozo, Braz. J. Chem. Eng.24, 3(2007)

6. Zh. G. Huang, H. Zhu and Q. P. Zhu, in Proc. IEEE Int. Computer Application and System Modeling (ICCASM'10 ), Taiyuan, China, (2010)

7. W. S. Wang, Cement, 11 (1990). In Chinese.

8. J. P. Pan, China Master Dissertations (Tsinghua University, Beijing, 2006). In Chinese.

9. S. J. Friedman, W. R. Marshal. Chem. Eng. Prog. 45, 8 (1949). 